\title{
Trente ans d'évacuateurs de crues au laboratoire
}

\section{Thirty years of laboratory study of spillways}

\author{
J. Gruat, C. Thirriot et D. Trivellato
}

Institut de Mécanique des Fluides de Toulouse

Laboratoire associé au CNRS

\section{Introduction}

Mafflus, pansus comme des pachydermes ou bien altiers comme un aigle, ils sont là, les évacuateurs de crue, au verrou de la gorge de la rivière ou à la clé de la grande nappe miroitante, à la fois garde-barrières et gendarmes de la circulation hydraulique.

Pour la plupart d'entre eux, leur vie est "despérément" vide et apparemment inutile.

Tels des sphynxs sous le soleil, leur grand corps, lourd, pose au destin la question de la statistique des extrêmes. Puis un beau jour ou plutôt un mauvais soir, tout s'explique, ils luttent contre des Niagaras, ils enserrent soudain dans leurs bras des fleuves de boues, ils sont cognés par de grands arbres déchirés agrippant leurs pieds dans la roche ou le béton, et violemment minés par l'eau en furie.

Mais quel paradoxe de voir ces œuvres pérennes, puissantes, massives et coûtant des fortunes, transformées en jouets maniables et éphémères au grè de la similitude dans les laboratoires d'hydraulique! Pourtant, c'est ainsi qu'ils naissent et prennent forme, ces grands ouvrages de la sauvegarde, comme nous l'ont patiemment appris quelques décennies d'étude sur maquettes au laboratoire d'Hydraulique de l'IMFT (Institut de Mécanique des Fluides de Toulouse).

Faisons donc un pélérinage dnas les archives du Laboratoire et dans le souvenir de nos Maîtres, les Professeurs Camichel et Escande, pour apprécier les possibilités et les limites de l'étude des évacuateurs de crue sur modèle réduit.

\section{Les évacuateurs de crue et leurs problèmes}

Un évacuateur de crue, comme son nom l'indique, a pour objet de laisser passer les débits exceptionnels dans les meilleures conditions.

La première de ces conditions étant de s'assurer que le passage de la crue n'entraine pas de submer- sion de l'ouvrage de retenue (surtout si celui-ci est en terre). Cette condition pourra être appréciée par la capacité d'évacuation représentée quantitativement par le graphe $Q\left(Z_{m}\right)$, avec $Q$ débit et $Z_{m}$ niveau d'eau à lamont proche. Ce graphe $Q\left(Z_{m}\right)$ rend compte globalement des performances hydrodynamiques de l'ouvrage.

Dans la plupart des 90 cas étudiés au cours des trente dernières années à l'Institut de Mécanique des Fluides de Toulouse, ce graphe $Q\left(Z_{m}\right)$ ne dépend pas des conditions de niveau d'eau à l'aval de l'ouvrage. Quelques rares exceptions concernent les seuils de retenue en rivière, libres de toutes vannes, qui, noyés sous les crues extrêmes, ne sont qu'une mince gêne à l'écoulement. A rapprocher aussi de cette circonstance, les barrages mobiles, à seul très bas, qui, vannes effacées, n'encombrent guère plus que des ponts.

Revenons donc à l'évacuateur de crue, protecteur de l'ouvrage de retenue. Si la submersion du barrage est évitée, les inondations à l'amont sont circonscrites dans un périmètre délimité par le remous. Nous n'en dirons pas plus pour l'influence de l'évacuateur de crue pour la sécurité de l'amont lointain.

Les conditions de sécurité à l'aval peuvent être examinées sous deux aspects:

- d'une part, l'atténuation du débit extrême d'une crue par l'étalement ou le laminage du volume d'eau de crue grâce aux concours conjugués de l'effort de stockage temporaire de la retenue et de l'effet de frein à l'écoulement dans le passage rétréci que constitue l'évacuateur,

- d'autre part, la défense des structures contre l'attaque de l'eau, soit les coups de boutoir violents mais francs, soit l'effet de mine sournois par les rouleaux et tourbillons au pied de l'ouvrage, à l'aval bien sûr, mais aussi parfois à l'amont (au bas des piles ou lorsque une partie des vannes est fermée).

Capacité d'évacuation et sécurité de l'ouvrage sont donc les deux questions essentielles que pose l'étude 
d'un évacuateur de crues, étude qui va être nuancée par le type de l'évacuateur.

Dans un essai de classification, on peut distinguer les évacuateurs suivant la hauteur de chute entre niveau amont $Z_{m}$ et niveau aval $Z_{v}$. Cette distinction physique qui prend en compte implicitement l'énergie à dissiper est toute relative et dépendra beaucoup de la qualité géologique et mécanique des terrains qui reçoivent l'eau à la sortie de l'évacuateur. On pourra parler de haute chute, de basse chute et de la catégorie intermédiaire, pas trop bien définie, de moyenne chute.

On peut aussi envisager un classement suivant que l'évacuation se fait toujours à l'air libre ou en puits.

Un autre critère de distinction important est l'existence de parties mobiles (vannes, barrages gonflables) qui peut aller jusqu'à l'effacement presque total du barrage au passage de la crue dans un équipement de basse chute.

Cette diversité de critère de classement montre déjà qu'il n'y a pas en matière d'évacuateur de crue de solutions stéréotypées et c'est ce qui conforte l'intérêt de l'étude particulière sur modèles réduits. Mais, en gros, dans la plupart des ouvrages d'évacuations, on retrouve les éléments suivants :

- un déversoir amont droit, frontal, latéral, circulaire pour les puits (qui peut être parfois remplacé ou complé. té par des vannes de fond).

- un canal d'évacuation à forte pente appelé souvent "coursier" lorsqu'il est suffisamment long (ouvrage de moyenne et haute chute). Il peut se terminer par un rétrécissement ou un relèvement du fond ou du radier pour créer un jet ou une nappe d'eau dont l'énergie sera partiellement dissipée par frottement dans l'air.

- un dispositif de réception aval dont la fonction est de dissiper l'énergie cinétique résiduelle de l'eau (qui est pratiquement toute l'énergie due à la chute dans le cas des chutes basses). On parle souvent de fosse d'amortissement naturelle (lorsque le jet liquide creuse un trou où il se love en tourbillon) ou artificielle (lorsque lhomme veut circonscrire la dissipation d'énergie). Mais dans les deux cas, à l'équilibre de configuration, l'énergie est dissipée au sein de la masse liquide. Avant l'équilibre, le trop plein d'énergie s'emploie rageusement à creuser sa niche par l'érosion et le transport de matériau. Et là, on pressent que la durée de la crue va intervenir de manière déterminante.

Devant la multiplicité des particularités, nous renoncerons donc à étiqueter de manière trop rigide les évacuateurs étudiés sur modèle à l'IMFT mais nous retiendrons les problèmes communs pas forcément rencontrés tous ensemble :

- la cnnnaissance de la capacité d'évacuation ou du coefficient de débit (dont il reste à préciser les conditions de définition),

- les champs de pression dans l'espace et dans le temps pour connaitre les efforts et la fatigue sur les parois,

- les phénomènes d'instabilités et d'onde,

- l'importance du frottement dans l'air et son entrâ̂nement,

- la dissipation d'énergie et l'érosion à la restitution, - les circonstances transitoires ou variables causées par l'évolution du chantier ou la variation du débit au cours de la crue.

\section{La compétition entre l'ordinateur et le mo- dèle réduit}

N'est-ce pas un baroud d'honneur que livrent les modèles réduits face aux énormes possibilités des ordinateurs de la dernière génération au fonctionnement "vectorisé"? Mais dans cette compétition, qui est David, qui est Goliath? D'un côté, l'intelligence spéculative enfouie dans les neurones électroniques et de l'autre, l'intelligence physique et concrète du plénomène naturel ou stimulé étalé au grand jour et qui parait devenir un jeu d'enfant.

On peut rappeler les exploits des ordinateurs dans la simulation des écoulements. Par exemple:

- la figuration de la houle déferlant sur une plage ou se brisant contre les digues avec éclatement de paquets d'eau comme le montre au magnétoscope un Institut de Calcul américain

- la circulation du sodium dans la cuve de Phénix étudiée par Sogreah à Grenoble

- les simulations de bassins portuaires examinées par le Centre d'Etude et de Recherche de Chatou.

Evidemment, dès qu'un programme assez universel existe, l'ordinateur devient imbattable sur le plan du délai: plus de souci de construction de maquettes, plus de congés de maladie, la multiplication des essais, l'édition quasi automatique du rapport d'étude. Bien sûr, la souplesse des éléments finis permet de représenter des parois aux formes compliquées. Mais les écoulements dans les évacuateurs aux particularités nombreuses sont "fortement" tridimensionnels et la finesse de découpage exigée entraîne des temps et des coût de calcul encore effarants ainsi que des matériels aux capacités de mémoires rapides et énormes. Et puis, l'ordinateur, même gigantesque, n'est rien sans le programme qui exige une équipe patiemment expérimentée.

Mais il y a plus, l'ordinateur a besoin de lois physiques explicitées analytiquement alors que le modèle les prend en compte globalement implicitement. il en prend d'ailleurs parfois trop en compte avec la capillarité. A contrario, des effets d'air sont sous estimés sur modèle. Mais ceux-ci sont les servitudes des effets d'échelle dans la similiłude.

\section{La similitude en sol majeur}

Dans les études d'évacuateurs deux types de phénomènes se présentent:

- les phénomènes hydrodynamiques prépondérants dans les écoulements et

- les phénomènes hydromécaniques régissant en particulier l'érosion des sols.

La similitude est assez bien maîtrisée du côté hydro. dynamique. Elle reste floue pour ce qui concerne ce phénomène majeur que sont le creusement du sol et l'entrainement des matériaux.

Il n'est pas question de ressasser ici un cours sur la similitude. Chacun sait qu'à partir de l'inventaire des $p$ grandeurs physiques ou paramètres intervenant dans le phénomène, l'analyse dimensionnelle permet de définir $p-3$ groupements sans dimensions qui doivent 
être conservés en passant de l'ouvrage grandeur nature ou prototype au modèle réduit (et réciproquement).

Bien sûr, il y a lieu de rassembler certains paramètres variables dans les familles de grandeurs que sont les fonctions. Comme les fonctions "êtres mathématiques" ne peuvent travailler que sur d'autres "êtres mathématiques" qui sont les nombres, ces fonctions ne doivent donc pas être déformées dans la transposition "modèleprototype". Ce postulat étant établi, il faudra donc veiller à la conservation des valeurs homologues "prototype et modèle" prises par les fonctions en un point particulier du site ou encore des nombres ayant même structure que ces fonctions.

Ceci peut paraître un galimatias esotérique inopportun pour parler de choses aussi concrètes que les modèles réduits. Passons donc à l'illustration et l'utilisation pragmatique.

Quelles sont les données physiques?

1. La description de l'évacuateur de crue par sa taille (une dimension de longueur $\mathrm{L}$ ) et sa forme (une multitude de rapports de dimensions de longueur conservés dans la similitude géométrique pure et par conséquent nous n'en parlerons pas).

2. La description du fluide par la donnée des paramètres:

- les masses volumiques $\rho$ pour l'eau, $\rho_{a}$. pour l'air, - les viscosités dynamiques $\eta$ pour l'eau, $\eta_{a}$ pour l'air, - $\sigma$ tension interfaciale en présence d'un fluide de référence qui est implicitement l'air.

3. Les caractéristiques rhéologiques du sol:

- $\rho_{\mathrm{s}}$ la masse volumique

$-\tau_{0}$ le seuil du taux de cisaillement assurant la dégradation et la caractéristique de la cohésion

- $d$ la taille caractéristique des particules

- $F(d)$ la fonction de répartition des tailles de particules

\section{L'indication des actions extérieures:}

- $g$ l'accélération de la pesanteur,

- $p_{a}$ la pression atmosphérique omniprésente dans les écoulements à surface libre.

\section{Les conditions de l'écoulement :}

- les variables explicatives, les niveaux $Z_{m}$ à l'amont et $Z_{v}$ à l'aval ou le débit $Q$ et le niveau $Z_{v}$,

- les variables dépendantes ou expliquées,

- les distributions de vitesse $V$ et dépression $P$.

Par application de l'analyse dimensionnelle, on va pouvoir construire les produits sans dimension dont le nombre dépendra du nombre de paramètres physiques retenus, nombre qui lui-même dépendra de la partie de l'écoulement étudié (par exemple:

- pour le déversoir amont, inutile de tenir compte de $Z_{v}$, et pour un bassin de tranquillisation en béton, pas besoin de parler de $\tau_{0}$, etc.).
On trouvera ainsi pêle-mêle :

- un nombre de Reynolds

$$
R e=\rho \frac{V L}{\eta} \quad \text { ou } \quad R e=\rho \frac{Q}{L \eta}
$$

caractéristique de l'importance relative des effets d'inertie et de viscosité ;

- un nombre de Froude

$$
F r=\frac{V}{\sqrt{g L}} \text { ou } \quad F r=\frac{Q}{L^{2} \sqrt{g L}}
$$

caractéristique du rapport de l'énergie cinétique et des travaux des forces de pesanteur;

- des nombres d'Euler

$$
E_{u}=\frac{P}{\rho V^{2}} \quad \text { ou } \quad E_{u}=\frac{P L^{4}}{\rho \mathrm{Q}^{2}}
$$

-- un nombre de Weber

$$
W e=\frac{\rho V^{2} L}{\sigma} \quad \text { ou } \quad W e=\frac{\rho Q^{2}}{\sigma L^{3}}
$$

caractéristique du rapport des forces d'inertie et des forces de capillarité ;

- des nombres caractéristiques de l'érosion, par exemple :

$$
\frac{\rho_{\mathrm{s}}}{\rho} \text { et } \frac{\tau_{0}}{\left(\rho_{\mathrm{s}}-\rho\right) g D}
$$

- des nombres caractéristiques de l'influence de l'air en plus des nombres de Weber et d'Euler:

$$
\rho_{\mathrm{a}} / \rho \text { et } \eta_{\mathrm{a}} / \eta
$$

et bien d'autres encore qui pourraient être obtenus par panachage ou combinaison des nombres ci-dessus.

Dans les écoulements à surface libre, les effets de la pesanteur sont essentiels, d'où la nécessité de conserver impérativement le nombre de Froude, ce qui induit à parler de similitude de Froude. Et souvent, on oublie toutes les autres contraintes! Parce qu'on ne peut pas y satisfaire en même temps.

Examinons rapidement les distorsions ainsi tolérées sur les principaux nombres caractéristiques.

Nous admettons que la similitude géométrique est assurée avec une échelle de longueur :

$$
\lambda_{\mathrm{L}}=\frac{L_{m}}{L_{p}}
$$

où $m$ désigne les grandeurs modèles

$p$ les grandeurs prototypes

Cette échelle est aussi celle des variations de pression.

Dans les études réalisées au laboratoire, $\lambda$ varie dans la fourchette de $1 / 200$ à 1 avec une fréquence prépondérante autour de $1 / 50$.

La conservation du nombre de Froude conduit:

- à une échelle de vitesse : $\lambda_{v}=\lambda_{L}^{1 / 2}$

- à une échelle de débit : $\lambda_{Q}=\lambda_{L}^{5 / 2}$

et à une échelle de temps: $\lambda_{t}=\lambda_{L}^{1 / 2}=\lambda_{v}$ 


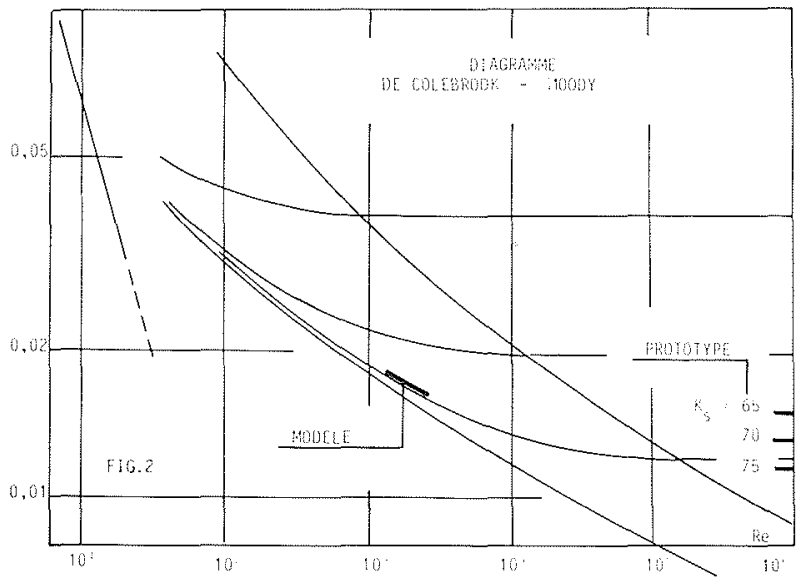

Figure 1 - Diagramme de Colebrook-Moody - Conditions d'écoulement sur le modèle et le prototype.

\section{Distorsion du nombre de Reynolds}

En similitude de Froude:

$$
\lambda_{R e}=\lambda^{3 / 2} \cdot \frac{\rho_{m}}{\rho_{p}} \cdot \frac{\eta_{p}}{\eta_{m}}
$$

En général, le fluide est le même sur modèle et prototype, c'est l'eau.

Pour assurer la conservation du nombre de Reynolds, il faudrait faire couler du mercure sur le modèle pour rapprocher $\lambda_{R e}$ de l'unité. Fort heureusement, la diminution du nombre de Reynolds sur le modèle n'a pas de conséquence catastrophique tant qu'on ne change pas de régime d'écoulement. Mais avec une échelle courant de $1 / 50$, le nombre de Reynolds est divisé par 350 et l'écoulement peut devenir laminaire dans certaines zones du modèle. La conséquence est une aggravation sensible des effets de pertes de charge. Quelques exemples de correspondance prototype-modèle ont été portés sur l'abaque de Colebrook de la figure 1 . Mais comme le plus souvent l'écoulement sur prototype est turbulent franchement rugueux, on ajuste la dissipation d'énergie comme sur le modèle de Golfech en plaçant des éléments créant des tourbillons dissipateurs (figure 2). En somme, on discrétise le problème tout comme en calcul numérique. D'autres fois, on augmente la rugosité du fond en plaçant du grillage ou du métal déployé [4] (Fig. 3).

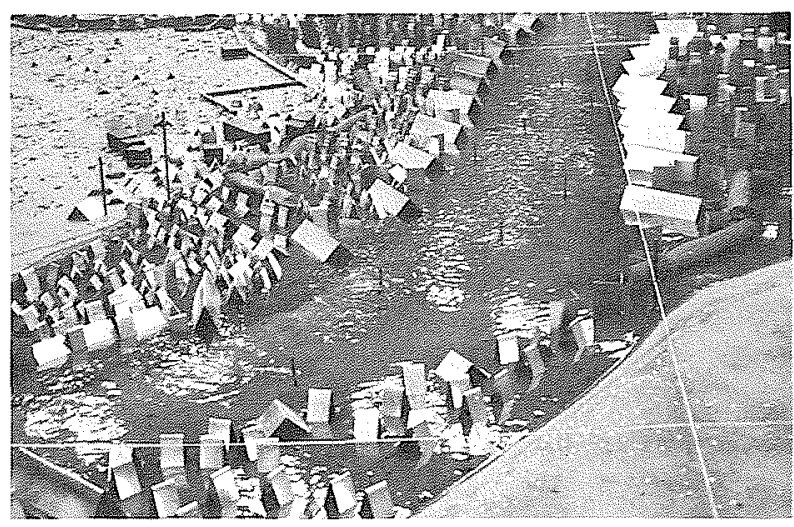

Figure 2 - Modèle de Golfech
Ce calibrage des pertes de charge est délicat et profite beaucoup de l'expérience accumulée par les hommes familiers du modèle réduit.

\section{Distorsion du nombre d'Euler}

Le nombre d'Euler sera estimé à partir de la pression de l'air, c'est-à-dire la pression ambiante.

Son échelle est donc:

$$
\lambda_{E u}=\lambda_{p a} \lambda_{L}^{-1}
$$

Si on ne prévoit aucun artifice, la pression atmosphérique est la même sur modèle et prototype et la distorsion est donc $\lambda_{L}^{-1}$.

Pour faire bien, il faudrait travailler sous vide partiel sur modèle. Les conditions économiques ne s'y prêtent pas mais l'influence de la distorsion sur Eu est en général négligeable.

\section{Distorsion du nombre de Weber}

Les effets capillaires marqués par l'inverse du nombre de Weber sont plus importants sur modèle que sur ouvrage. Mais, dans la réalité du modèle réduit comme

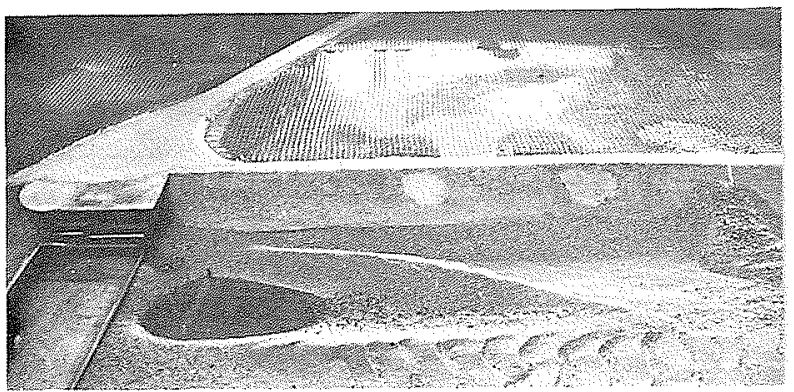

Figure 3 - Adjonction de grillage ou de métal déployé pour augmenter la rugosité du fond du modèle

du prototype, ces effets ne sont pas purs. Ils sont très sensibles à la mouillabilité des parois - une plaque de plastique grasse, une paroi de béton encombrée de poussière vont être non mouillables à l'eau et tout change - la flaque qui, sur modèle, représente l'onde d'inondation refuse de s'étendre et forme un bourrelet l'addition de produits tensio-actifs comme ce fut fait dans le champ d'inondation de Golfech arrange très simplement la situation.

Plus importante est la conséquence sur la structure des jets. La tension superficielle protège les jets de l'éclatement en gouttes et de la dispersion avec frottement dans les couches d'air. Ainsi, sur le modèle, la dissipation énergétique dans les nappes et jets à la sortie des cuillères, déviateurs et sauts de ski est relativement plus faible que sur le prototype. On pourrait envisager d'examiner l'influence du réglagle de la tension superficielle sur l'effet d'échelle encore par emploi de tensioactifs. Avec la rupture des tubes d'écoulements, on retrouve les bulles et gouttes, arguments des Journées S.H.F. 1962.

\section{Distorsion du nombre caractéristique de l'érosion}

La cohésion d'un matériau est difficile à cerner avec précision. Elle peut être estimée de manière floue 
par le biais de la vitesse de début d'érosion $V_{0}$ telle que

$$
\tau_{0} \sim \rho V_{0}^{2}
$$

Des essais sur des mélanges d'argile et de sable ont été effectués à l'Ecole Nationale d'Ingénieurs de Tunis en vue de régler la cohésion à la similitude.

Pour maintenir la constance du nombre caractéristique

$$
\tau_{0} / g D\left(\rho_{s}-\rho\right)
$$

différentes voies pourront être envisagées.

Sauf pour des lits naturels constitués de gros blocs, généralement on ne respectera pas la similitude géométrique pour les matériaux de fond. On devra donc réaliser:

$$
\frac{\lambda_{v_{0}}^{2}}{\lambda_{D}} \cdot \frac{\rho_{s_{p}}-\rho}{\rho_{s_{m}}-\rho}=1
$$

Comme $\lambda_{D}>\lambda_{L}$ et que $\lambda_{v_{0}}^{2} \cong \lambda_{L}$, il faut assurer $\rho_{s_{m}}<\rho_{s_{p}}$. On prendra donc des matériaux plus légers que le sable ou les galets tels des noyaux d'abricots broyés (formule jadis utilisée à Chatou) ou des particules plastiques (solution couteuse si le volume du fond affouillable est important). Au laboratoire, on a aussi utilisé le charbon (fines de houille) et du bois dur traité.

\section{Similitude distordue géométriquement}

Nous venons de voir que l'on admettait une échelle de taille des particules différentes de l'échelle de longueur. Ceci introduit une distorsion sur l'échelle des aspérités de parois. C'est l'amorce de la distorsion de l'échelle des hauteurs par rapport à l'échelle des longueurs. Pour ne pas trop réduire le nombre de Reynolds, on peut en effet envisager de prendre une échelle $\lambda_{H}$ des hauteurs différente de l'échelle géométrique $\lambda_{L}$ afférente aux distances en plan. La similitude de Froude conduit alors à :

$$
\begin{array}{ll}
\lambda_{V}=\lambda_{H}^{1 / 2} & \lambda_{T}=\lambda_{L} \lambda_{H}^{-1 / 2} \\
\lambda_{Q}=\lambda_{H}^{3 / 2} \lambda_{L} &
\end{array}
$$

Mais dans le cas des évacuateurs de crue, il faut user de cet artifice avec précaution parce qu'avec une très forte distorsion $\delta=\lambda_{H} / \lambda_{L}$, on va transformer un seuil épais de coefficient de débit $C_{q} \cong 0,38$ en un seuil mince de coefficient de débit $C_{q} \cong 0,45$.

Dans un modèle représentant de larges zones, le pis aller consiste à utiliser la distorsion partout sauf pour l'évacuateur de crue qui obéira à l'échelle $\lambda_{H}$ en similitude géométrique.

En conclusion de ce cheminement raccourci à travers la similitude, on voit que l'analyse dimensionnelle met en accusation le modèle réduit sur plusieurs points dont notamment les effets liés à l'entraînement d'air et les phénomènes d'érosion.

\section{L'hydrodynamique et la capacité d'évacuation}

La capacité d'évacuation est essentiellement réglée par l'écoulement au dessus du déversoir situé en tête

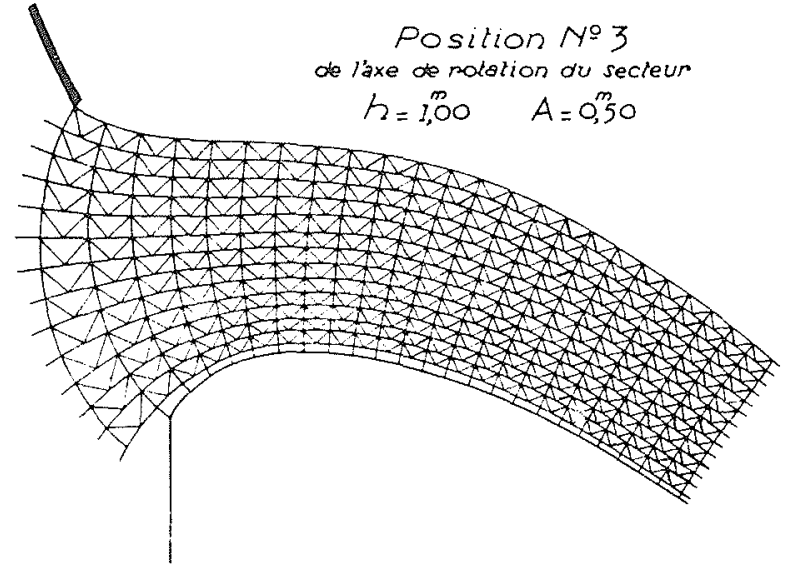

Figure 4-Construction du Prasil - Capacité d'évacuation d'un évacuateur d'après L. Escande

de l'évacuateur. Et c'est là le domaine privilégié de la similitude de Froude, parce qu'en mouvement accéléré et sur une petite distance, la dissipation d'énergie est insignifiante et la vitesse pas encore assez élevée pour que les effets de l'air jouent un rôle de distorsion.

Bien sûr, on peut rétorquer que le calcul peut lui aussi fournir de bons résultats dans cette zone. Témoin, la bonne concordance obtenue par L. Escande entre mesure et construction de Prasil pour un seuil plan surmonté d'une vanne (figure 4) qui fait penser à l'aménagement de Ferrières en Ariège.

Mais nous l'avons déjà dit, l'écoulement est souvent fortement tridimensionnel: c'est bien le cas du bec de canard de Yasmos (figures 5 et 6 ).

Le modèle réduit donne alors de manière élégante, sûre et rapide, le graphe $Q(H), H$ étant la charge audessus de la crête du seuil.

Les figures 7 et 8 donnent des exemples de tels graphes à partir desquels on peut essayer de définir l'évolution d'un coefficient hybride de débit en se rapportant conventionnellement à la définition pour un seuil simple:

$$
Q=C_{q} B H \sqrt{2 g H}
$$

avec $B$ longueur développée le long de la crête du déversoir.

Il y aurait long à dire sur l'hydrodynamique à l'amont immédiat du seuil déversant et surtout à l'aval, sur la

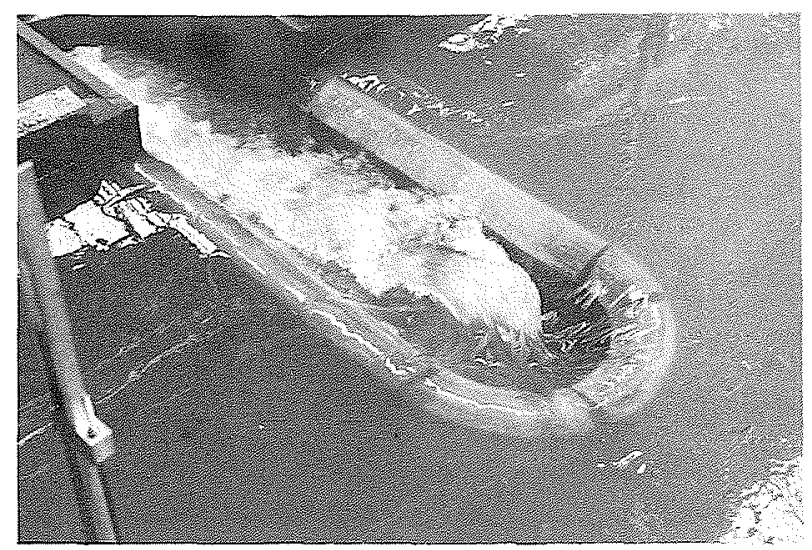

Figure 5 - Evacuateur en bec de canard de Yasmos (Grèce) 


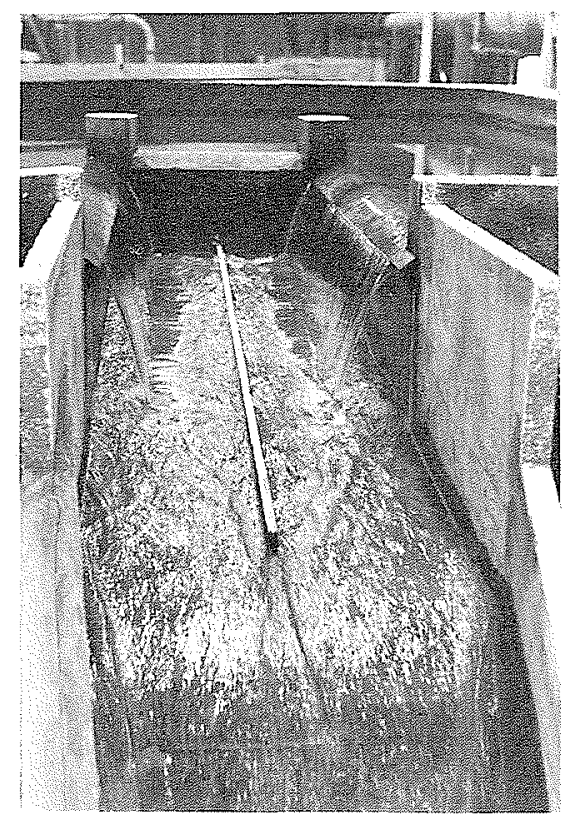

Figure 6 - Evacuateur en bec de canard de Yasmos (Grèce)

position des épis qui redresent l'écoulement face au seuil, sur l'élimination des crêtes de coqs dans le canal d'évacuation sur la stabilisation par l'entrée d'air ou par la fente aspiratrice bienfaisante proposée par L. Escande, sur les retouches des modèles des profils des seuils.

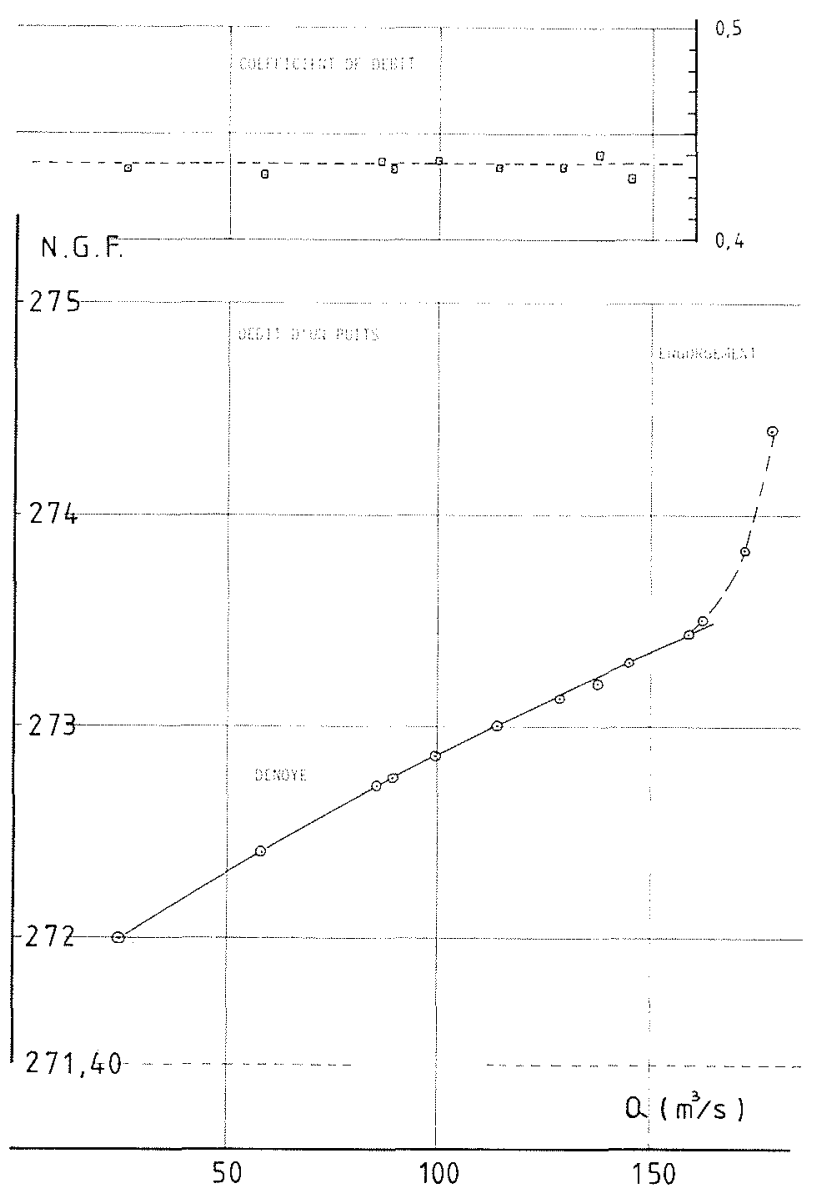

Figure 7 - Débit d'un puits en $\mathrm{m}^{3} / \mathrm{s}$ en fonction de la hauteur au-dessus de la crête du seuil

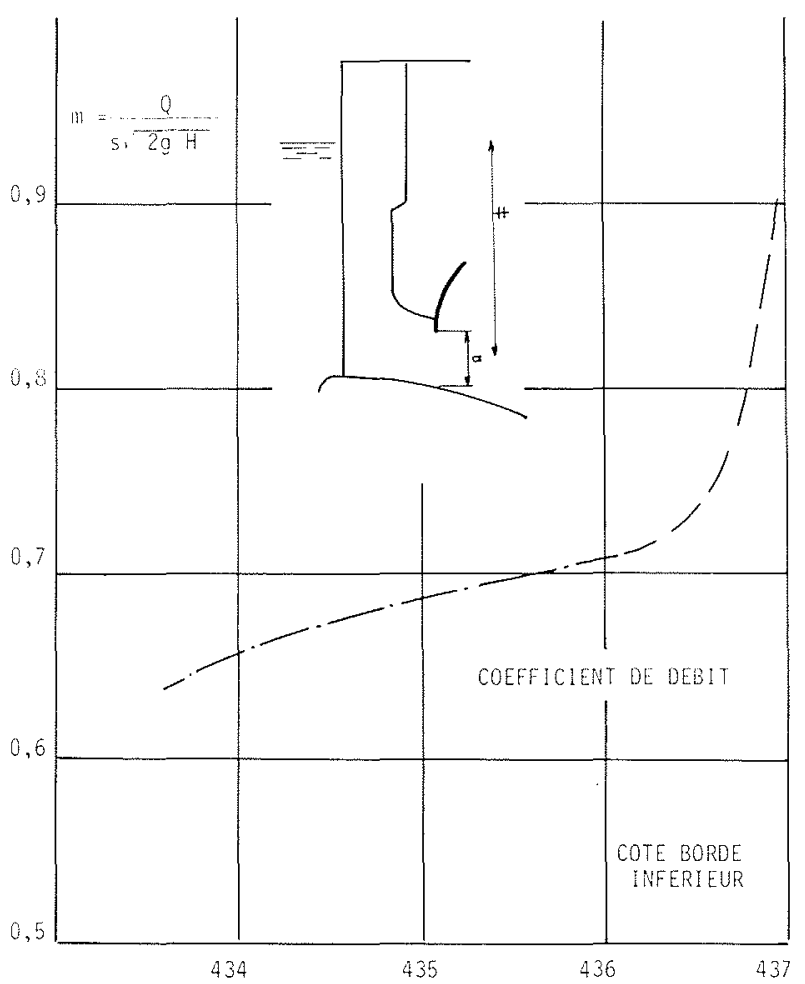

Figure 8 - Coefficient de débit en fonction de la hauteur audessus de la crête du seuil

Celui qui a un peu pratiqué le modèle réduit connaît les caractères capricieux de l'hydraulique des ouvrages courts. Une petite modification produit ici une amélioration, plus loin une détérioration comme sur le modèle d'El Mali où la suppression d'un petit angle vif sur la cuillère a modifié forme, structure et impact du jet. Il s'agit constamment de compromis en ayant toujours à l'esprit la facilité de réalisation sur le chantier, le coût et la sécurité. C'est ce dernier point qui est surveillé dans l'étude des instabilités qui pourrait apparaître dans l'écoulement simultané par vanne de fond et sur clapet tel l'ouvrage de Ferrières. L. Escande (encore lui!) a mené sur ce problème une très belle étude sur modèle réduit déterminant avec précision la période des battements qu'il explique par la naissance, le développement et l'entraînement de deux tourbillons antagonistes aux bords du panneau central.

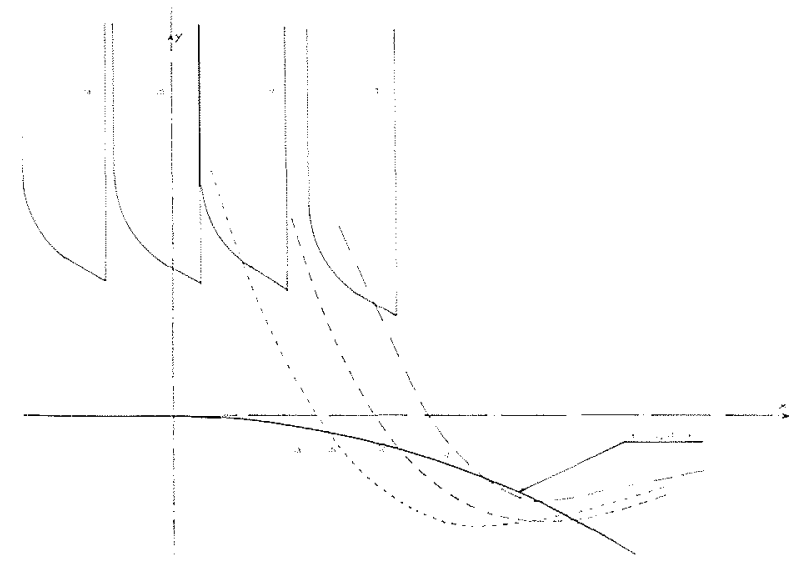

Figure 9 - Evacuateur de Ferrières - Répartition des pressions le long d'un seuil déversant -- Influence de la position du tablier 


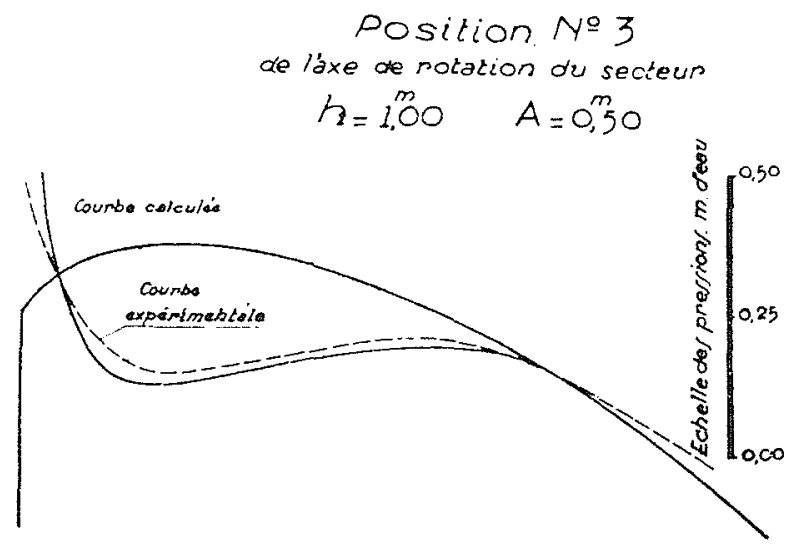

Figure 10 - Répartition des pressions le long du seuil versant, d'après L. Escande

Ces rouleaux peuvent faire penser aux allées de tourbillons de Von Karman derrière un cylindre dans un écoulement en charge.

\section{La répartition des pressions dans l'espace et dans le temps}

L'exemple de l'évacuateur de Ferrières est bien démonstratif de l'importance de l'étude de la distribution des pressions le long d'un seuil déversant (figure 9). L'extension de la zone de dépression dépend de la position de la plaque. L. Escande avait lui-même fait une étude systématique et très approfondie de l'influence de l'implantation d'une vanne secteur, aussi bien théoriquement qu'expérimentalement, pour différentes ouvertures et différents débits. La figure 10 donne un exemple de ses résultats.

Quelques années plus tard, en 1958, dans son ouvrage "Barrages mobiles et prises d'eau en rivière", M. Bouvard insiste aussi sur le choix de la position d'une vanne de fond. Il propose de la placer à une distance de la crête du seuil Creager $d=0,20 H_{0}$ vers l'aval, $H_{0}$ étant la charge nominale. On évite ainsi le décollement aux petits débits suivant la parabole de chute libre.
La tension supportée par le parement du déversoir est gênante mais souvent elle est aggravée par des fluctuations de pression qui créent une fatigue du matériau. La figure 11 donne une idée des fluctuations observées sur le modèle réduit de Stratos. Bien sûr, il faut être prudent pour la transposition sur prototype; en effet ces variations apparemment aléatoires peuvent être expliquées pour partie par la macroturbulence et pour partie par les phénomènes d'entrainement d'air (qui stimulent aussi la turbulence). Or, nous avons vu que ces deux phénomènes ne suivaient pas parfaitement la similitude de Froude. Peut-être pourrait-on envisager, dans la mise à l'échelle sur prototype, d'utiliser comme guide correcteur l'intensité de la vitesse turbulente conformément au raisonnement suivant : admettons que les fluctuations de pression $\left|p^{\prime}\right|$ soient proportionnelles à $|v|^{2}, v$ étant en un point l'écart type des fluctuations de vitesse dans le temps. Admettons aussi que la dissipation d'énergie soit proportionnelle à $|v|^{2}$. Comme fil conducteur, utilisons la formule universelle de perte de charge établie pour les tuyaux :

$$
\Delta H=f \frac{\widetilde{V}^{2}}{2 g} \frac{\Delta L}{\phi}=C \frac{|v|^{2}}{2 g} \frac{\Delta L}{\phi} .
$$

formule dans laquelle

$f$ : est un coefficient universel de perte de charge donné par l'abaque de Colebrook-Moody

$\phi$ : une dimension transversale généralisation du dia

mètre

$\widetilde{V}$ : vitesse moyenne

Alors: $\quad\left|p^{\prime}\right| \sim f \widetilde{V}^{2}$

L'échelle des fluctuations de pression serait ainsi:

$$
\lambda_{\left|p^{\prime}\right|}=\lambda_{L} \cdot \frac{f_{m}}{f_{p}}
$$

Pour fixer les idées, utilisons la formule de Blasius valable pour l'écoulement turbulent lisse :

$$
f=\left(\frac{1}{100 \mathrm{Re}}\right)^{1 / 4}
$$

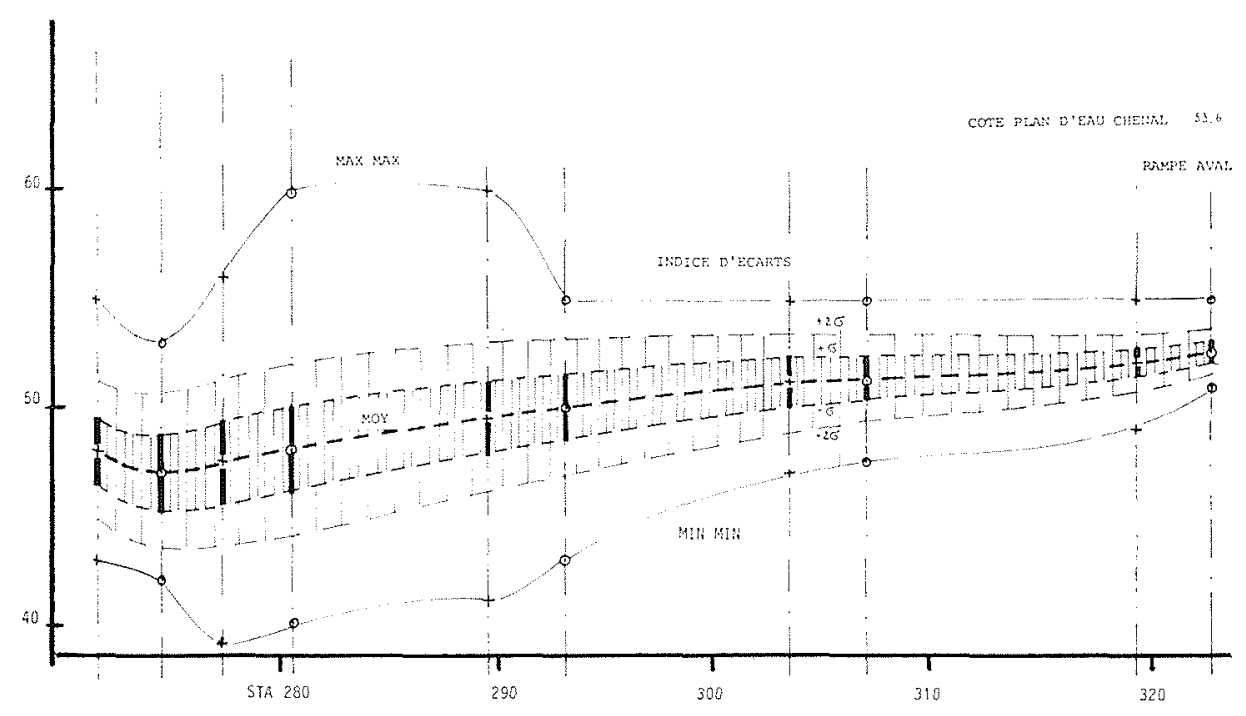

Figure 11 - Fluctuations des pressions sur le modèle de Stratos (Grèce) 


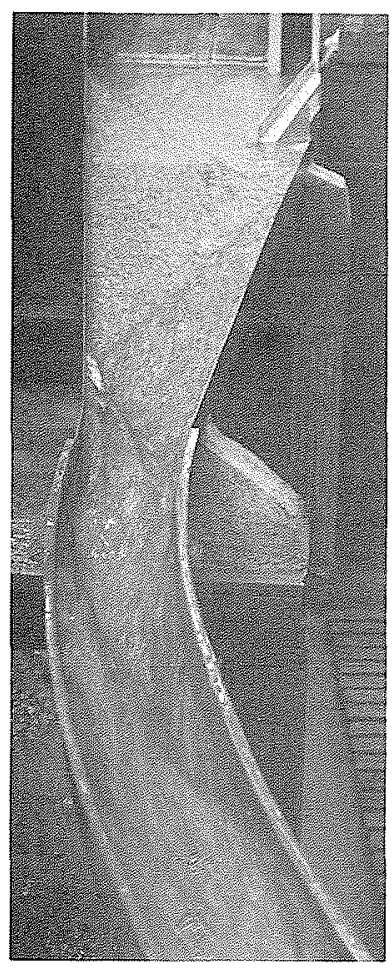

Figure 12 - Modèle de St-Alban de Villars - Réfléchissement des ondes stationnaires sur les parois verticales

Alors: $\quad \lambda_{\left|p^{\prime}\right|}=\lambda_{L} \cdot\left(\frac{R e_{p}}{R e_{m}}\right)^{1 / 4}=\lambda_{L}^{5 / 8}$

Les fluctuations de pression sur le prototype seraient donc moins importantes que ne le laisserait prévoir la transposition pure et simple suivant la similitude de Froude ce qui va heureusement dans le sens de la sécurité.

\section{Les coursiers et les ondes stationnaires}

Dans les écoulement torrentiels, il apparaît des ondes stationnaires qui se réfléchissent sur les parois comme le montre la figure 12 réalisée sur le modèle de SaintAlban de Villards. La figure 13 donne une idée des surélévations obtenues. On voit que ce phénomène exige un rehaussement très important des bajoyers du coursier. Dans la recherche des solutions possibles, on va d'abord jouer sur la genèse des ondes stationnaires en modifiant les formes et en particulier les angles, le devers. On peut aussi jouer sur la pente.

Une formule plus sophistiquée consiste à passer en écoulement fluvial à l'aide d'un ressaut dans la zone d'amorçage des ondes stationnaires puis à retrouver l'écoulement torrentiel graduellement par l'augmentation progressive de la pente.

Dans le cas de Saint Alban de Villards, le projeteur a même demandé l'examen de deux formes de radier: le radier lisse qui entraîne de très fortes surélévations d'onde comme nous l'avons vu sur les figures 12 et 13 et le radier rugueux créé par blocs de $30 \mathrm{~cm}$ rejointés au béton qui conduit au total à un tirant d'eau moyen beaucoup plus grand mais avec des ondes stationnaires fortement émoussées.

\section{La dissipation d'énergie et l'érosion}

A la sortie de l'évacuateur, l'écoulement se présente en jet noyé ou dans l'air, compact ou étalé en nappe. Si le jet, par le truchement d'un saut de ski, d'une cuillère ou du relèvement du radier, fait un trajet dans l'air il va perdre une partic de son énergie cinétique par frottement dans l'air mais de toutes façons, comme pour le jet noyé, la fin de la dissipation d'énergie ou tranquillisation va se passer dans la fosse naturelle ou

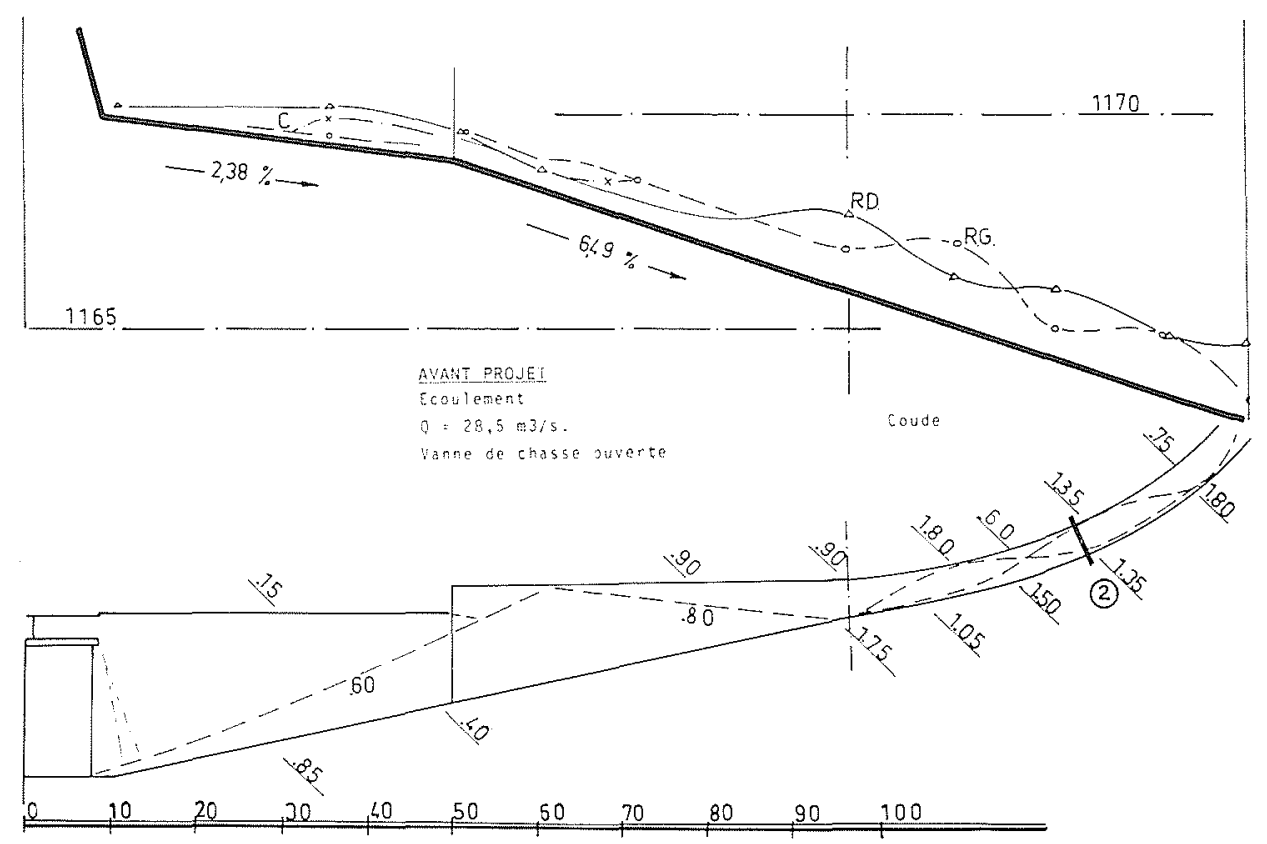

Figure 13 - Modèle de St-Alban de Villars - Surélévation des bajoyers pour contenir les ondes stationnaires 


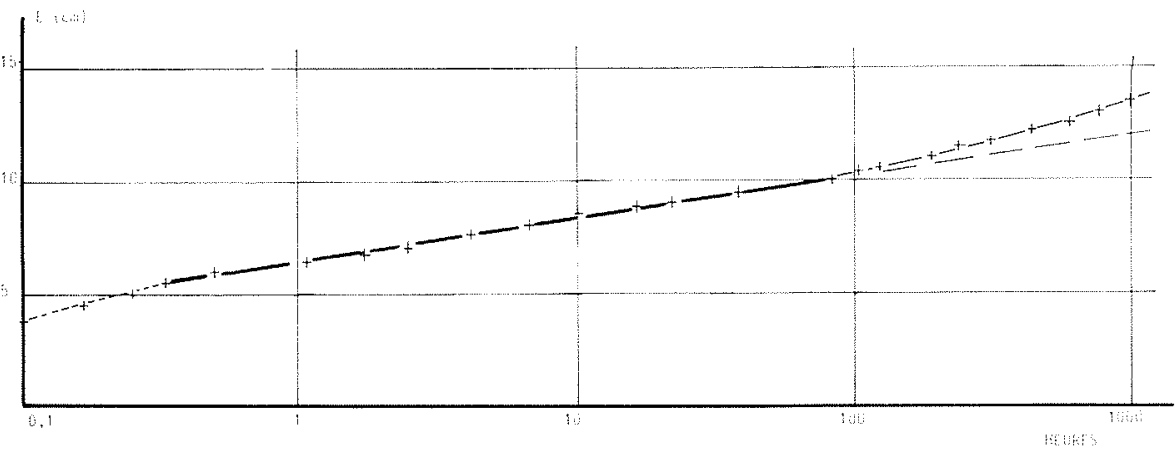

Figure 14 - Schéma de l'affouillement - Profondeur de la fosse en fonction du temps, d'après J. Pichon

construite à l'aval de l'évacuateur. Cette dissipation va se passer pour partie dans le travail d'arrachement des particules du sol et pour partie dans la masse liquide agitée par le jet. La répartition entre travail de transport solide et agitation turbulente va évoluer tout au long des premières longues et fortes crues. Beaucoup de travail de creusement au début, rien que de la dissipation turbulente à la fin lorsque le jet a creusé sa niche dans le fond de la rivière ou de la vallée. La durée de la crue va donc être décisive. Cependant, la plupart du temps, on considère des durées suffisamment longues pour supposer que l'affouillement ultérieur sera négligeable. Mais, en réalité, l'érosion continue insidieusement. De nombreux travaux sur modèles réduits ont été effectués au laboratoire pour juger de l'évolution des dimensions de la fosse affouillée et de la

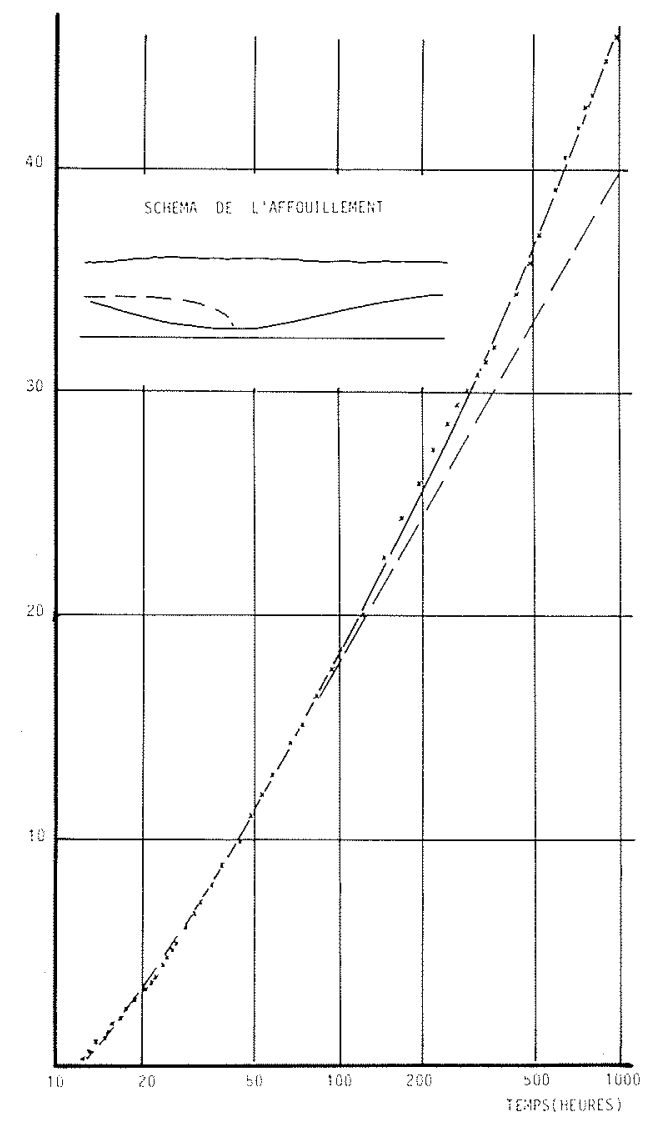

Figure 15 - Schéma de l'affouillement - Poids de sable évacué en fonction du temps, d'après J. Pichon masse de matériaux emportés (Bourrec, Pichon 1967, Kazemi-Moud 1970).

Les figures 14 et 15 montrent les résultats obtenus au cours d'un essai de 1000 heures à l'aval d'un radier plan pour un sable à $d_{90}=3,3 \mathrm{~mm}$.

Schoklitsch a été l'un des premiers, sinon le premier, à étudier ce phénomène de creusement. Il a proposé une loi puissance pour l'évaluation de la profondeur $E$ de la fosse:

$$
E=A t^{1 / 6} H^{1 / 2} q^{1 / 3}+2,15 a
$$

où $t$ est la durée

$H$ la charge

$q$ le débit par unité de largeur

a la profondeur de la surface de l'eau à l'aval sous le niveau de la surface du radier.

Cette formule est convenable pour représenter le début du phénomène mais inadéquate pour l'évolution à très long terme qui serait plutôt logarithmique comme l'ont montré les essais de l'IMFT.

Compte tenu de toutes les contraintes et de toutes les critiques possibles de la similitude de l'érosion, on peut dire :

- que les essais sur modèle réduit donnent un ordre de grandeur des paramètres de l'affouillement

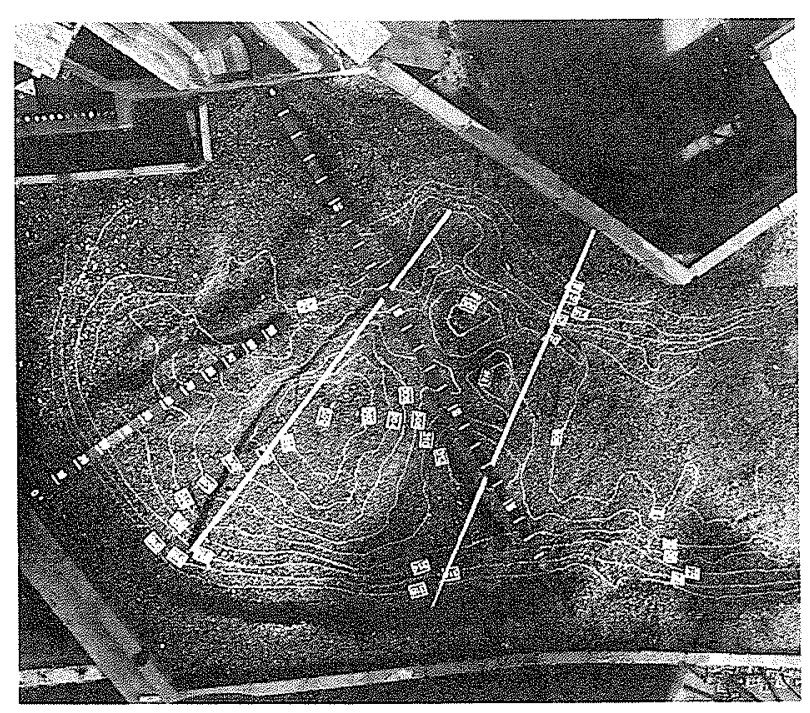

Figure 16 - Evacuateur de crues - Saut de ski - Résultats des affouillements pour un débit de $1600 \mathrm{~m}^{3} / \mathrm{s}$. 


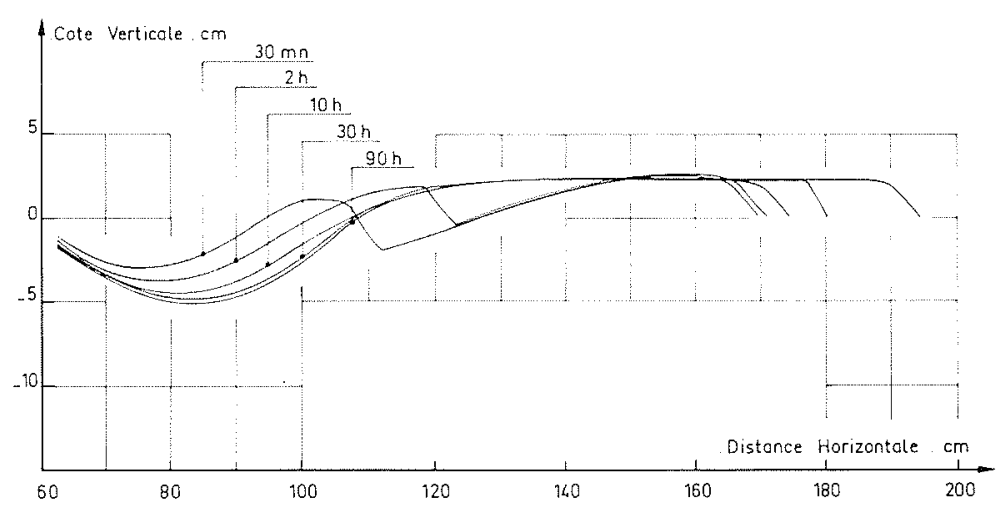

Figure 17 - Relevé de la fosse d'affouillement, d'après Kazemi

- mais qu'ils fournissent d'excellentes informations qualitatives par exemple sur l'explication des formes d'érosion et la localisation des rouleaux.

Et n'est-il pas spectaculaire de voir apparaître après le bouillonnement opaque de la fosse, les abysses dessinées par les fils de laine déposés suivant les lignes de niveau à la vidange progressive du modèle (Fig. 16). Cette topographie révèle parfois la surprise de plusieurs creux, résultat de la compétition de plusieus rouleaux cachés sous le jet ombellifère. Encore plus simplifié à deux dimensions, en canal vitré, le modèle réduit permet aussi de comprendre la progression de la barre aval qui ferme la fosse, à la manière d'une dune (Fig. 17).

L'exploitation des essais sur modèle réduit est essentiellement fondée sur le postulat raisonnable suivant: dans la nature, les effets de l'érosion afférents à diverses solutions de projet et construction seront classés dans le mème ordre que sur le modèle. Plus schématiquement, la meilleure solution sur le modèle, parmi plusieurs, restera la meilleure dans la nature. Ceci a pu être confirmé par des essais en similitude avec des variations d'échelle modérées.

\section{L'entrainement d'air}

Nous avons déjà parlé de l'influence de l'air à propos de la distorsion du nombre de Weber et du nombre d'Euler. Devant l'utopie d'une similitude parfaite, examinons les conséquences de la similitude de Froude pragmatique.

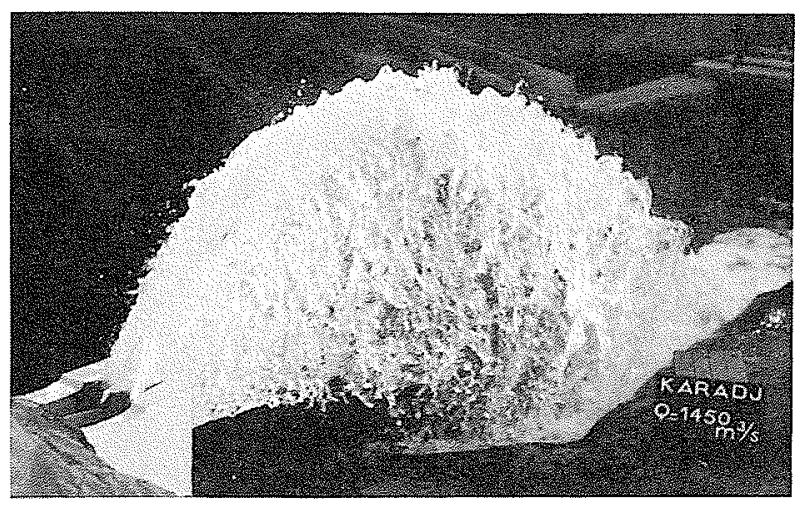

Figure 18 - Modèle de Karadj - Gonflement des écoulements à la sortie du saut de ski
Les effets de l'entraînement d'air vont dans le bon sens de la sécurité, quand on passe du modèle au prototype, pour ce qui concerne la dissipation de l'énergie (comme dans le jet de Karadj, par exemple, figure 18), l'atténuation des ondes en écoulement supercritique (mais resterait à revoir de manière critique les conditions transcritiques). Par contre, ils vont dans le mauvais sens en provoquant :

- un gonflement des écoulements "in situ" qui nécessite une surélévation des bajoyers,

- un débit d'air dont il faut tenir compte dans les galeries d'évacuation en régime permanent ou transitoire (fig. 19)

- et une distorsion appréciable dans l'amorçage des siphons existants sur certains évacuateurs de crue.

Cet air entraîné provoque aussi des oscillations, qui peuvent aller jusqu'à des battements éreintants pour l'ouvrage. M. Varlet n'a-t-il pas observé "sur un vieux barrage de trois à quatre mètres de hauteur, à coursier très raide, que les vibrations de la nappe ébranlaient l'atmosphère, qu'à plusieurs centaines de mètres on
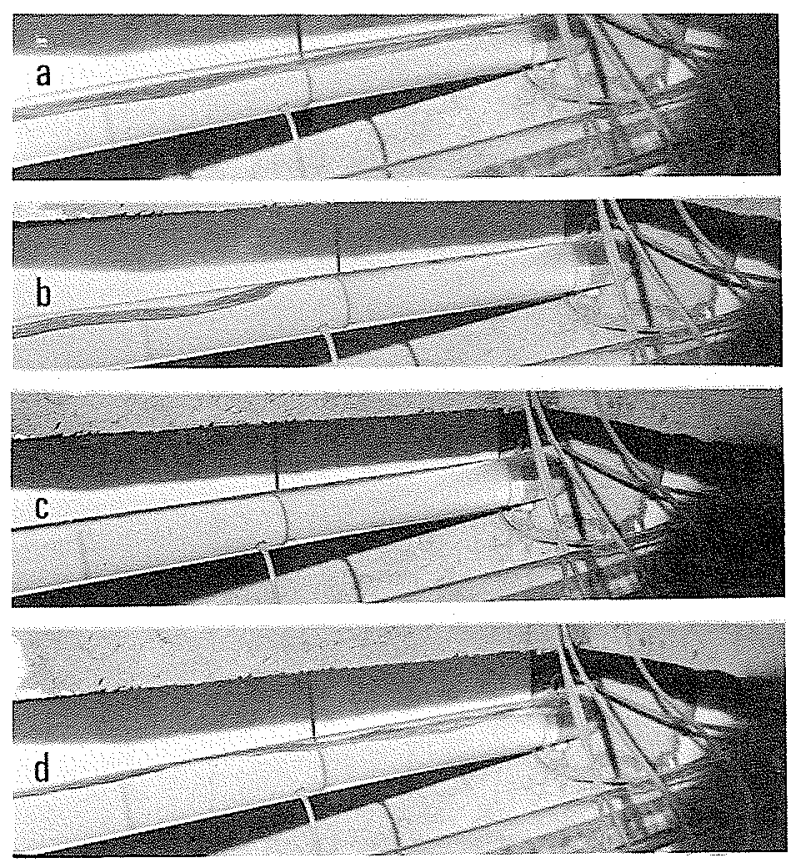

Figure 19 - Galerie d'évacuation

a) écoulement à surface libre c) écoulement à pleine section b) onde positive d'amont d) onde négative d'amont 
entendait un frémissement régulier qui accompagnait les battements périodiques et impressionnants de la lame déversante". Pour éviter ces inconvénients, L. Escande proposa le système de la fente aspiratrice, stabilisant confortablement la nappe déversante tout en augmentant le coefficient de débit.

Cet entraînement d'air devient une affaire politique devant les Syndicats de pêcheurs qui prêchent pour la défense des aloses contre l'eau blanche des échelles à poissons. En effet, ces éléments annexes des barrages déversoirs connaissent un regain d'intérêt avec la focalisation sur la qualité de la vie des hommes et des poissons et notre laboratoire a eu à étudier maints modèles réduits sur les échelles à poissons (tout dernièrement l'ouvrage de Bergerac). On pourrait croire, en suivant les arpèges gracieux ou turbulents de la truite, que l'air est une bonne chose pour le poisson. Bien sûr, mais à dose homéopathique en solution et non point en brassées de bulles. Quel sera donc le devenir de ces bulles happées vers le fond par la turbulence, rappelées vers le soleil par Archimède, ligotées par la capillarité?

\section{Le fonctionnement hydraulique aux dif- férentes étapes de la construction}

Le modèle réduit est un conseiller discret mais avisé pour le projeteur, le maître d'œuvre et le chef de chantier soucieux des aléas hydrologiques lors de la construction de l'ouvrage. Dans un monde lilliputien, dans un temps rétréci, on peut prévoir les caprices du fleuve grossi par orage soudain et obligé de s'engouffrer dans la galerie de dérivation provisoire (qui restera peut-être évacuateur de fond) ou bien de contoumer le batardeau protégeant quelques passes en construction.

Un exemple récent, étudié au laboratoire, est celui de l'ouvrage marocain de Ait Youb.

\section{L'évacuateur face aux débits variés}

Quand on parle d'évacuation de crues on pense aux débits énormes pour des périodes de retour de 10000 ans par exemple. Sur une durée de vie de quelques petites centaines d'années, la probabilité, pour le prototype, de vivre l'évènement, vu sur le modèle, est donc tout de même très faible. Mais il y a également les crues, plus fréquentes, qui doivent passer dans des conditions convenables.

Un saut de ski assurera très bien l'éloignement du trou d'érosion au débit de 10000 ans, et laissera tomber piteusement le débit séculaire au pied de l'ouvrage. Il est donc nécessaire que cela se passe sans risque. D'où la recherche, sur modèle réduit, de l'adaptation aux débits moyens, de la solution trouvée pour les débits gigantesques $\left(\right.$ giga $\left.=10^{9}\right)$.

Un exemple récemment étudié est celui de l'aménagement de Plan d'Arem où, pour les débits modérés, un ressaut était localisé sur le coursier dissipant heureusement l'énergie, à fort débit ce ressaut était balayé vers l'aval, puis expulsé.

Le fonctionnement, à débit intermédiaire, peut ne pas exiger la mobilisation de toutes les passes d'évacuation. Du seul point de vue de la capacité de décharge des seuils déversants amont, on peut envisager la fermeture d'une partie des vannes, mais l'hydrodynamique peut en être radicalement modifiće. Ce fut par exemple le cas sur le modèle de Karadj, ouvrage de haute chute, où l'ouverture d'une seule des deux vannes donnait naissance à un jet qui en se réfléchissant furieusement provoquait au saut de ski un pincement défavorable. La solution consistat donc en la mise en cellule individuelle des écoulements à la sortie de chaque vanne. Toujours pour le modèle de Karadj, à la sortie de la tuyère, la solution proposée était convenable: pas de matelas d'eau et un écoulement suffisamment viril à tous débits.

Un bon exemple, d'étude sur modèle, de la conjugaison des ouvertures de vannes fut celui de l'ouvrage d'Ait Youb déjà cité.

\section{Conclusion}

Depuis qu'Archimède faisait joujou dans sa baignoire, les modèles réduits ont acquis, peut être pas leurs lettres de noblesse mais bien leurs lettres de vieillesse avec son sens ancestral de la sagesse et de l'expérience. Car la pratique du modèle réduit est une lente éducation qui ne peut être transmise qu'à la manière socratique du cotoiement quotidien. Et les hommes de notre génération ont essayé de grapiller en la matière, un peu de l'énorme expérience d'experts tels Léopold Escande et Louis Castex.

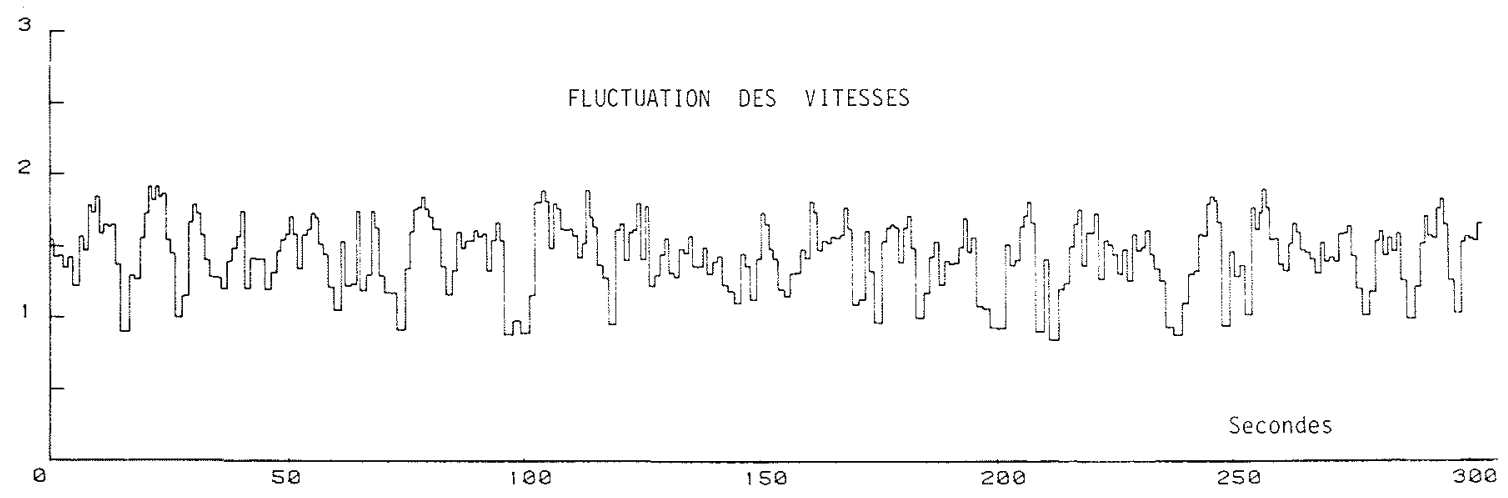

Figure 20 - Fluctuations des vitesses dans l'échelle à poissons de Bergerac 
Mais le modèle réduit n'est pas figé dans une technique désuète. Il sait profiter du progrès technologique dans le domaine de la mesure, de la transmission et du traitement de l'information. Il y a déjà plus de seize ans que fonctionne, au laboratoire, le pilotage du modèle réduit par ordinateur (Manou-Dournes). Bien sûr, notre expérience est modeste actuellement face à celles des grands laboratoires de Chatou, de Sogreah et de Maison Alfort, mais elle est constamment et utilement mise à contribution même dans des études de détail comme celle des fluctuations de vitesse dans l'échelle à poisson de Bergerac (Fig. 20). Et il y a plus, car le modèle réduit, parce qu'il résoud implicitement les équations de l'hydrodynamique, est un calculateur analogique. Grâce à sa supériorité modeste dans l'étude des écoulements tridimensionnels, il peut former en association avec un ordinateur, un calculateur hybride qui étudiera, lui, les grandes extensions, inacessibles au modèle, sous peine d'effet d'échelle parasite.

Face au problème de l'évacuation des crues, les modèles réduits nous apparaissent comme un outil privilégié mais ils sont évidemment tributaires de la statistique des extrêmes pour l'ampleur du débit à examiner. Un modèle réduit peut faire le beau temps d'un laboratoire mais il ne peut faire la pluie dans la nature.

Ainsi, lhomme du modèle réduit est-il l'homme du dialogue: avec le théoricien de la mécanique des fluides, pour critiquer ou affiner la similitude, avec l'infomaticien, pour du calcul hybride et le traitement de l'information, avec l'hydrologue des statistiques et enfin avec le bâtisseur d'ouvrage dont il partage le pragmatisme, le souci de la sécurité et les préoccupations économiques.

\section{Bibliographie}

BOUVARD M. - 1958, Barrages mobiles et prise d'eau en rivière. Eyrolles, 243 pages.

CAMICHEL C., ESCANDE L. - 1938, Similitude hydrodynamique et technique des modèles réduits. Publication Scientifique et Technique du Ministère de l'Air, $\mathrm{n}^{\circ} 127$.

Congrès International Des Grands Barrages XIII. - 1979, Congrès de New Delhi, Inde.

CREAJER W., JUSTIN J. - 1950, Hydroelectric Handbook. John Wiley and Sons, New-York.

ESCANDE L. - 1929, Etude théorique et expérience sur la similitude des fluides incompressibles pesants. Edition de la Revue Générale l'Electricité, Paris.

ESCANDE L. - 1951, Compléments d'hydraulique - Deuxième partie. Dunod, pp. 188 à 229 .

GHETTI. - 1955, Similitude des affouillements du lit des rivières dans les zones de diffusion de veines rapides. Etudes et Recherches $\mathrm{n}^{\circ} 169$, Institut de l'Université de Padoue.

GINOCCHIO R. - 1959, Aménagements hydroélectriques. Eyrolles, 480 pages.

KAZEMI-MOUD M. - 1969, Etude expérimentale des affouillements à l'aval d'un seuil déversant en fonction du temps. Thèse de Docteur d'Université - Faculté des Sciences de Toulouse.

PICHON J. - 1967, Contribution à l'étude des affouillements à l'aval d'un seuil déversant. Thèse de Docteur-Ingénieur Faculté des Sciences de Toulouse.

SCHOKLITSCH. - 1951, Calcul des fosses d'affouillement à l'aval d'un ouvrage de retenue. Wasser und EnergieWirtschaft, $n^{\circ} 2$.

S.H.F. - 1962, Bulles et Gouttes. Comptes Rendus des $7^{\circ}$ Journées de l'Hydraulique Société Hydrotechnique de France, Paris.

VARLET. - 1958, Aménagement, utilisation et prix de revient des usines hydrauliques. Eyrolles.

VARLET. - 1965, Usine de dérivation. Eyrolles.
M. le Président. - Je remercie M. le Professeur GRUAT de sa communication extrêmement intéressante, résumant 30 années d'activités d'un Laboratoire connu dans le monde entier; ainsi qu'on a pu le constater au cours de l'exposé, une part importante des études - un tiers environ- a porté sur des ouvrages étrangers.

J'ouvre maintenant la discussion.

M. POCHET. - Une dépression de $2,50 \mathrm{~m}$ sur un radier, à l'aval d'une vanne, est-elle dangereuse ?

$M$. GRUAT. - Oui, parce que cela représente $2,5 \mathrm{t}$ par $\mathrm{m}^{2}$ de radier, ce qui peut soulever la dalle par sous-pression ou arracher le revêtement recouvrant le radier.

M. Le Président. - M. GRUAT nous a indiqué que le modèle réduit ne pouvait pas donner l'affouillement limite à l'aval d'un évacuateur, des matériaux continuant à être entraînés par l'eau évacuée et ce phénomène n'ayant pas de limite. Or, très souvent les rapports relatifs aux essais sur modèles définissent l'affouillement limite, dans l'échelle des temps adoptée bien entendu. Je souhaiterais que M. GRUAT précise ce point particulier.
M. GRUAT. - Il ne faut pas perdre de vue, à ce sujet, le phénomène de l'érosion à l'aval du barrage par les eaux évacuées non chargées, les matériaux s'étant déposés dans la retenue. Il s'agit là, certes, d'un phénomène très complexe, qui se manifeste de façon continue dans le temps.

Mais, il faut cependant reconnaître que des essais sur modèle, qui représentent une durée équivalant à la période de retour de la crue de projet -10000 ans par exemple-, donnent une limite maximale pour l'érosion, qui ne sera jamais atteinte dans la réalité, et donc une marge de sécurité satisfaisante.

$M$. le Président. - S'il n'y a plus d'autres questions, je vais à nouveau remercier, M. GRUAT et toute l'équipe du Laboratoire de Toulouse, notamment MM. THIRRIOT et TRIVELLATO, qui ont participé à la préparation de cette très intéressante communication.

Les deux communications qui vont suivre sont des monographies d'ouvrages qui ont posé des problèmes originaux. A ce titre, elles sont très intéressantes. Je passe la parole à $M$. STERENBERG, Ingénieur en Chef au Bureau d'IngénieursConseils COYNE et BELLIER, pour la présentation de sa communication. 